\title{
Preparation of Blood Products Grouping Reagent
}

National Cancer Institute

\section{Source}

National Cancer Institute. Preparation of Blood Products Grouping Reagent. NCI

Thesaurus. Code C112941.

A procedure to isolate blood group antigens for use in serological testing. 